\title{
Probing Big Bang Nucleosynthesis deep underground
}

\author{
Carlo Gustavino*广 \\ INFN, Sezione di Roma, Piazzale A. Moro 2, 00185 Roma, Italy \\ E-mail: carlo.gustavinoeromal.infn.it
}

The Big Bang Nucleosynthesis (BBN) theory allows to calculate the abundance of isotopes created in the early universe. Interestingly, the primordial abundance of deuterium deduced from observations of pristine gas at high redshifts is more accurate with respect to the computed value because the BBN calculation is affected by the paucity of data for the deuterium-burning reaction $D(p, \gamma)^{3} \mathrm{He}$. In fact, only a single dataset is currently available in the BBN energy range, in which the authors state systematic error of $9 \%$. The concern for the $D(p, \gamma)^{3} \mathrm{He}$ cross section error is made worse by the fact that the theoretical and experimental values do not agree at the level of $20 \%$. A new measurement is presently in progress at the LUNA (Laboratory for Underground Nuclear astrophysics) accelerator, operating deep underground at the Gran Sasso Laboratory, Italy. The main goal is the study of the $D(p, \gamma)^{3} \mathrm{He}$ cross section in the BBN energy range with accuracy. The LUNA measurement is described and preliminary results are discussed and compared with ab-initio calculations. The impact of this measurement in cosmology and particle physics is also highlighted. In particular, a precision measurement allows to derive the universal baryon density $\Omega_{b}$ with accuracy comparable to the one obtained by the PLANCK experiment. Finally, the accurate knowledge of the $D(p, \gamma)^{3} \mathrm{He}$ cross section increases the sensitivity to probe the existence of relativistic particles (e.g. sterile neutrinos, hot axions etc.) not foreseen in the standard model.

The 26th International Nuclear Physics Conference

11-16 September, 2016

Adelaide, Australia

\footnotetext{
* Speaker.

${ }^{\dagger}$ for the LUNA collaboration.
} 


\section{Big Bang Nucleosynthesis}

Big Bang Nucleosynthesis (BBN) occurs in the first minutes of cosmic time, as result of the competition between the expansion of the Universe and the nuclear reaction rates. The expansion rate of the universe is governed by the Freidmann equation:

$$
H^{2}=\frac{8 \pi}{3} G \rho
$$

Were $H$ is the Hubble parameter, $G$ is the Newton's gravitational constant and $\rho$ is the energy density which, in the early Universe, is dominated by the "radiation", i.e. the contributions from massless or extremely relativistic particles. The radiation density is often expressed as follows:

$$
\rho=\rho_{\gamma}\left(1+\frac{7}{8}\left(\frac{4}{11}\right)^{4 / 3} N_{e f f}\right)
$$

In this formula $\rho_{\gamma}$ is the photon density and $N_{e f f}$ is the contribution of other relativistic species. Using this formula $N_{\text {eff }}=3.046$ if only the three known neutrino families are considered. Figure 1 shows the leading processes of the BBN chain. At the end of BBN, nearly all the free neutrons end up bound into ${ }^{4} \mathrm{He}$ nuclei, while all the other isotopes are present in residual quantities. The only free parameter to calculate the primordial abundances in standard BBN is the baryon density $\Omega_{b}$, which is usually expressed normalized to the black-body photon density as $\eta=\Omega_{b} / n_{\gamma}$. Constraints on cosmology and particle physics can be obtained by comparing BBN calculated abundances with astronomical observations of light isotopes $\left({ }^{2} \mathrm{H},{ }^{3} \mathrm{He},{ }^{4} \mathrm{He},{ }^{6} \mathrm{Li},{ }^{7} \mathrm{Li}\right)$ in specific astrophysical sites. Therefore, in order to derive stringent constraints on cosmology and particle physics, are very important both the accuracy of astronomical observations and the precise knowledge of nuclear cross sections of BBN leading processes.

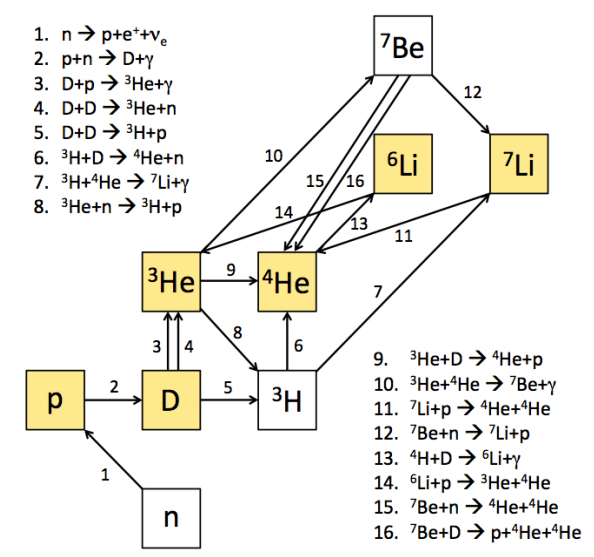

Figure 1: Leading processes of Big Bang Nucleosynthesis. Yellow boxes mark stable isotopes.

\section{Nuclear Astrophysics}

At energies of interest in astroparticle physics $(0.01 \div 1 \mathrm{MeV})$ the cross-section $\sigma(E)$ drops almost exponentially with decreasing energy $E$, due to the repulsion of charged nuclei. For this 
reason, in nuclear astrophysics the nuclear cross section $\sigma(E)$ is often factorized as follows:

$$
\sigma(E)=\frac{S(E) e^{-2 \pi \eta}}{E}
$$

In this formula, the exponential term takes into account the Coulomb barrier, while the astrophysical factor $S(E)$ contains all the nuclear effects. The Sommerfeld parameter $\eta$ is given by $2 \pi \eta=31.29 Z_{1} Z_{2}(\mu / E)^{1 / 2} . Z_{1}$ and $Z_{2}$ are the nuclear charges of the interacting nuclei. $\mu$ is their reduced mass (in units of a.m.u.), and $E$ is the center of mass energy (in units of keV). Due to the low reaction yield, direct measurements at low energy are severely hampered by the background induced by cosmic rays. For this reason the LUNA collaboration carries out its measurements with the world's only underground accelerator facility, operating at the "Laboratori Nazionali del Gran Sasso" (LNGS). In fact, the ultra-low background at LNGS makes possible to study the nuclear reactions well below the Coulomb barrier.

\section{$2.1{ }^{3} \mathrm{He}(\alpha, \gamma)^{7}$ Be.}

The BBN production of ${ }^{7} \mathrm{Li}$ is dominated by the ${ }^{3} \mathrm{He}(\alpha, \gamma){ }^{7} \mathrm{Be}$ reaction, with subsequent decay of radioactive ${ }^{7} \mathrm{Be}$ to ${ }^{7} \mathrm{Li}$. The ${ }^{3} \mathrm{He}(\alpha, \gamma){ }^{7} \mathrm{Be}$ reaction was studied at LUNA using two different experimental techniques: by detecting the promptly emitted $\gamma$-rays from the reaction and by measuring the ${ }^{7} \mathrm{Be}$ activity created in the experiment. The setup consisted of a windowless ${ }^{3} \mathrm{He}$ gas target, a ${ }^{4} \mathrm{He}^{+}$beam and a high purity germanium detector (HPGe) to measure the prompt $\gamma$ 's yield. The ${ }^{7} \mathrm{Be}$ activity of the beam stopper were also measured with a HPGe detector installed underground. Figure 2 (left) shows the LUNA result and literature data [1]. Note that only the LUNA data are well inside the BBN energy region. The LUNA result exacerbates the "lithium problem", excluding a nuclear solution to solve the tension between theory and observations [1].
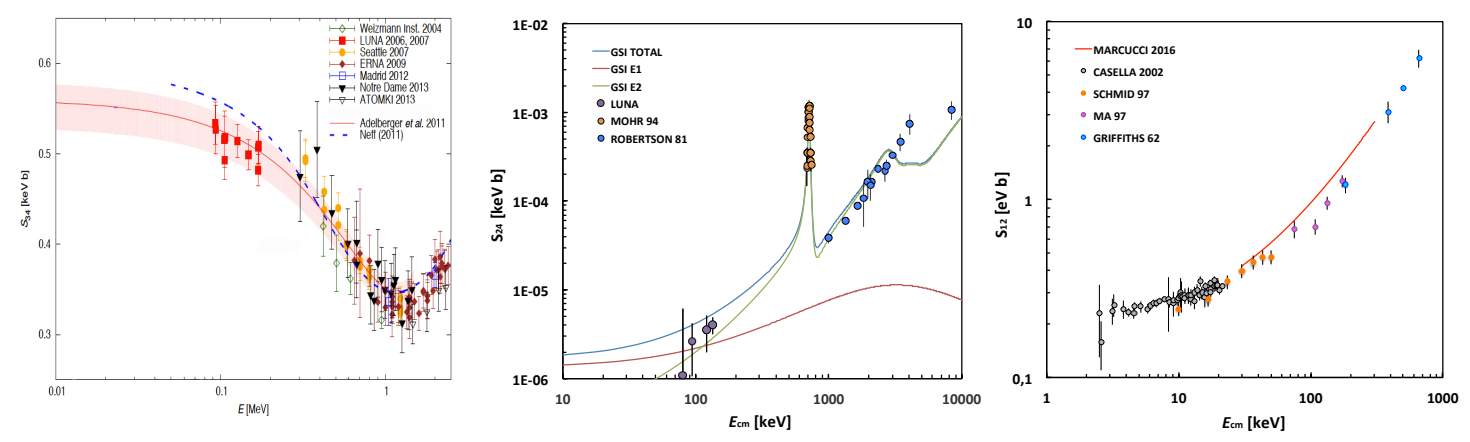

Figure 2: Left: astrophysical S-factor data of the ${ }^{3} \mathrm{He}(\alpha, \gamma)^{7} \mathrm{Be}$ reaction as a function of the center-of-mass energy. A theoretical curve and the ab initio prediction are also shown. Center: astrophysical S-factor data of the ${ }^{2} H(\alpha, \gamma)^{6}$ Li reaction as a function of the center-of-mass energy. The LUNA data are shown with all the previous direct measurements. The continuous lines show the theoretical E1, E2, and total $S_{24}$ factors describing recent Coulomb dissociation data. Right: S-factor data for the reaction ${ }^{2} H(p, \gamma)^{3} \mathrm{He}$. the red solid curve shows the prediction of recent ab initio theoretical calculation. See [1] and references therein. 


\section{$2.2{ }^{2} \mathbf{H}(\alpha, \gamma)^{6} \mathbf{L i}$.}

Standard BBN production of ${ }^{6} \mathrm{Li}$ is dominated by just one nuclear reaction, ${ }^{2} \mathrm{H}(\alpha, \gamma){ }^{6} \mathrm{Li}$. Before LUNA no direct measurements were performed inside the BBN energy region, making the BBN calculation concerning ${ }^{6} L i$ very uncertain. The ${ }^{2} \mathrm{H}(\alpha, \gamma)^{6} \mathrm{Li}$ S-factor has been recently measured at low energy by LUNA, with an $\alpha$ beam and a windowless deuterium gas target. The main problems for this experiment was the very low cross section (about $60 \mathrm{pb}$ at $E_{c m}=133 \mathrm{keV}$ ) and the existence of a significant beam induced background. Figure 2 (center) shows the LUNA result together with previous direct measurements [1,2] and theoretical calculations [3]. The direct LUNA measurement excludes a nuclear solution to explain the debated overabundance of this isotope in metal poor stars.

\section{$2.3{ }^{2} \mathbf{H}(\mathbf{p}, \gamma){ }^{3} \mathrm{He}$.}

As shown in figure 3, the deuterium abundance is very sensitive to $\eta$ ( $\eta=$ baryon-to-photon density ratio) and to $N_{\text {eff }}$. Presently the main obstacle to improve the sensitivity in deriving $\eta$ and/or $N_{\text {eff }}$ is the uncertainty of BBN calculation, while the primordial abundance obtained from observations of pristine gas at high redshifts have an uncertainty of about 1.5\% [4]. In particular

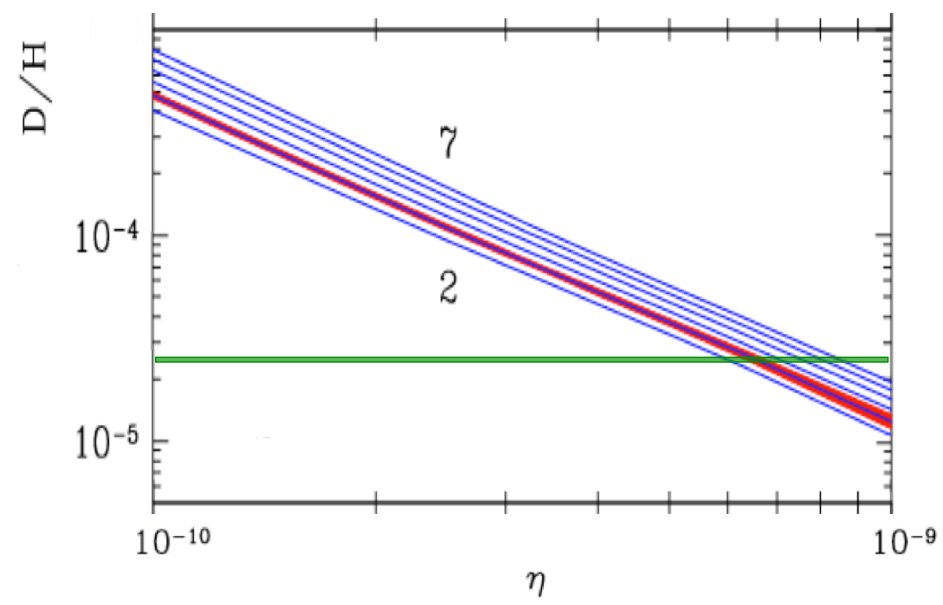

Figure 3: Deuterium abundance as functions of baryon-to-photon ratio. The blue lines indicate abundances for a single value (integer plus 0.046) of $N_{\text {eff }}$. The red bands indicate the nuclear uncertainty on those yields for $N_{\text {eff }}=3.046$. The green band indicates the observational uncertainty of $(\mathrm{D} / \mathrm{H})_{\text {obs }}$ [4].

the comparison of $(D / H)_{B B N}$ and $(D / H)_{o b s}$ provides $100 \Omega_{b, 0} h^{2}(B B N)=2.20 \pm 0.04 \pm 0.02$ [4]. In these equation $\Omega_{b, 0}$ is the present day baryon density of the universe and $h$ is the Hubble constant in units of $100 \mathrm{~km} \mathrm{~s}^{-1} \mathrm{Mpc}^{-1}$. The first uncertainty term is due to the BBN calculation, while the second one is due to the error of direct observations. The primordial abundance of deuterium depends on the four reactions shown in table 1. Its theoretical error is twice with respect to the uncertainty obtained by direct observations, and it is mainly due to the uncertainty of ${ }^{2} \mathrm{H}(\mathrm{p}, \gamma)^{3} \mathrm{He}$ cross section data [8]. The uncertainty due to this reaction is even larger if ab initio prediction is taken into account. Figure 2 (right) shows the data of the ${ }^{2} H(p, \gamma)^{3} \mathrm{He}$ reaction in literature. Note that only a single dataset of $S_{12}$ is currently available in the relevant BBN energy range, in 
Table 1: List of the leading reactions and corresponding rate symbols controlling the deuterium abundance after BBN. The last column shows the error on the ratio $D / H$ coming from the uncertainty in the cross section of each reaction, for a fixed baryon density $\Omega_{b} h^{2}=0.02207$ [8].

\begin{tabular}{|l|c|c|}
\hline Reaction & Rate Symbol & $\sigma_{D / H} \cdot 10^{5}$ \\
\hline \hline$p(n, \gamma)^{2} H$ & $R_{1}$ & \pm 0.002 \\
$d(p, \gamma)^{3} H e$ & $R_{2}$ & \pm 0.062 \\
$d(d, n)^{3} H e$ & $R_{3}$ & \pm 0.020 \\
$d(d, p)^{3} H$ & $R_{4}$ & \pm 0.0013 \\
\hline
\end{tabular}

which the authors state systematic uncertainty of 9\% [5]. However the fit of experimental data is about $20 \%$ lower than the theoretical calculation [6]. In this plot the LUNA data obtained with the previous $50 \mathrm{kV}$ pilot accelerator are also shown [7]. The present LUNA $400 \mathrm{kV}$ facility make possible to extend the measurements up to $E_{c m}=266 \mathrm{keV}$, i.e. well inside the BBN energy range. Figure 4 a) shows the scheme of the setup where a barrel BGO detector is implemented. The ongoing measurements are performed with the proton beam produced by the $400 \mathrm{kV}$ accelerator, a windowless deuterium gas target and a $4 \pi$ BGO crystal to detect prompt $\gamma_{\mathrm{s}}$ [7]. The study with the BGO detector will be accomplished by using a different layout in which a Germanium detector is faced to the gas target in a close geometry, as shown in figure $4 \mathrm{~b}$ ). With this configuration the angular distribution of photons emitted by the ${ }^{2} \mathrm{H}(p, \gamma)^{3} \mathrm{He}$ reaction can be inferred by exploiting the doppler effect affecting the energy of $\gamma$ 's produced along the beam line $E_{\gamma}$, as indicated by the following relationship:

$$
E_{\gamma}=\frac{m_{p}^{2}+m_{d}^{2}-m_{H e}^{2}+2 E_{p} m_{d}}{2\left(E_{p}+m_{d}^{2}-p_{p} \cos \theta\right)}
$$

In this formula, $m_{p}, m_{d}, m_{H e}$ are the proton, deuterium and ${ }^{3} H e$ masses. $E_{p}$ and $p_{p}$ are the energy and momentum of beam protons and $\theta$ is the angle of photons with respect to the beam line. Figure 5 shows the result of a preliminary measurement measurement performed with the HPGe detector. Note that the energy distribution of emitted photons is well in agreement with ab initio calculation (see figure caption).
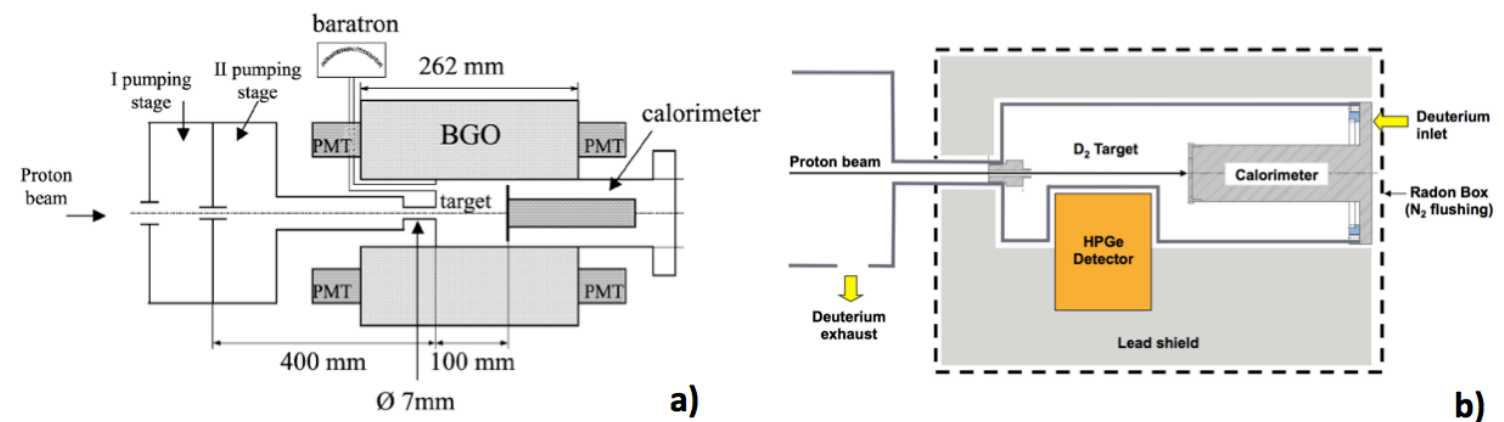

Figure 4: a): Scheme of gas target setup and BGO detector. b): Scheme of gas target setup and HPGe detector. 


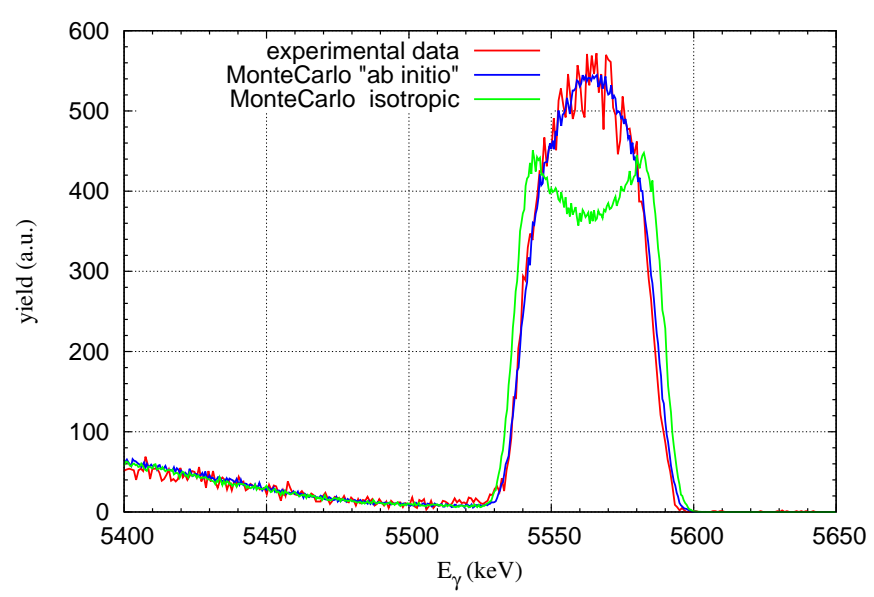

Figure 5: Simulated spectra of the ${ }^{2} H(p, \gamma)^{3} H e$ reaction, assuming isotropic (green) and ab initio (blue) angular distribution at $E_{c m}=112.5 \mathrm{keV}$. The experimental data (red) are also shown. Data have been normalized to remark the close agreement with the ab initio angular distribution.

\section{Conclusions}

The LUNA collaboration has measured with high accuracy several leading processes of BBN. The renewed study of the ${ }^{2} \mathrm{H}(\mathrm{p}, \gamma)^{3} \mathrm{He}$ reaction is motivated by the paucity of data at BBN energies of this reaction. The goal is to reach an accuracy at the $3 \%$ level, considerably better than the $9 \%$ uncertainty estimated in [5] and much lower than the $20 \%$ difference between data and nuclear calculations. The accurate study in the BBN energy range at the underground Gran Sasso laboratory will allow to considerably improve the determination of $\Omega_{b}$ and $N_{e f f}$, through the comparison of calculated abundance $(D / H)_{B B N}$ with the precise value of $(D / H)_{B B N}$ derived from observations in metal-poor damped Lyman-alpha systems observations.

\section{References}

[1] C. Gustavino et al, Eur. Phys. J. A (2016) 52, 74 (2016).

[2] M. Anders et al, PRL 113, 042501 (2014).

[3] F. Hammache et al, Phys. Rev. C 82, 065803 (2010).

[4] R. Cooke et al, ApJ. 781, 31, (2014).

[5] L. Ma et al, Phys. Rev. C 55, 588 (1997).

[6] L.E. Marcucci et al, PRL 116, 102501 (2016).

[7] C. Casella et al, Nuclear Physics A 706 203Đ216. (2002).

[8] E. Di Valentino et al, Phys. Rev. D 90, 023543 (2014). 